\title{
Craniocerebral Eumycetoma: A Rare Masquerader Of Meningioma
}

\author{
Dr. Mah Sheena K M *, Dr. Lilly Rajeevan", Dr. Shalini Kuruvila*, Dr. Kavitha K P * \\ * Department of Pathology, Aster MIMS, Calicut, Kerala. India.
}

DOI: 10.29322/IJSRP.10.02.2020.p9834

http://dx.doi.org/10.29322/IJSRP.10.02.2020.p9834

\begin{abstract}
Eumycetomas of craniocerebral are rare An extraordinary lesion in an extraordinary location in a 56-year-old male! We report here a case of eumycetoma involving brain and skull bone in a middle-aged male who presented with seizures and right sided weakness. Imaging showed a dural-based lesion enhancing moderately on contrast. It was mistaken for meningioma clinically and radiologically. Craniocerebral eumycetoma usually presents with lump on scalp and sinuses. To the best of our knowledge and belief, ours is one of the very few cases in the English Literature where a eumycetoma has presented as a mass lesion without discharging sinuses. It is imperative to keep such atypical features of an infective etiology in mind because they may be one of the differentials of "dural" based lesions where only a biopsy may suffice in the absence of significant mass effect to prove the diagnosis.
\end{abstract}

Index Terms- Craniocerebral, dural based, eumycetoma, meningioma.

\section{INTRODUCTION}

$\mathrm{M}$ ycetomas are common in tropical and subtropical areas. They are localized chronic, suppurative and deforming granulomatous infection. It is a disorder, characterized by a triad of localized swelling, underlying sinus tracts, and production of grains or granules affecting subcutaneous tissue, skin, and bones, mainly of feet $[1,2]$. Etiologically Mycetomas are of two types, Eumycetoma caused by fungus and actinomycetoma caused by bacteria. Many species of fungi and bacteria have been identified as causative agents. They enter into the skin usually following trauma. As the management of these two etiological types of Mycetomas are entirely different, even though difficult a definite diagnosis is essential after a proper histopathological and microbiological examination. Eventhough serological test exists they are not so reliable; however, molecular techniques to identify relevant antigens have shown promise [2]. We herewith report a patient with eumycotic mycetoma affecting the scalp, skull bone, dura and underlying brain parenchyma presenting with many atypical features.

\section{CASE REPORT}

A 53-year-old male presented in March 2017 with an episode of late-onset seizures. He was a known diabetic on treatment.He was evaluated for seizures.MRI brain plain with contrast done - showed a lesion in the right frontal region. The patient was put on follow up as he was not willing for surgery and started on antiepileptic. In the meantime, FNAC was considered which was not feasible as the bone over lesion was thick. During follow up patient presented with a similar episode of seizure in September 2017. Again an MRI was done which showed an increase in the size of the lesion. Since the patient was still not willing for surgery, he was advised to remain in close follow up. In October 2018 patient presented with progressive weakness of left upper and lower limb and difficulty in walking. General examination was normal. Glasgow coma scale score was 15/15 (E4 M6 V5) and pupils were equal and reacting to light. Power in left upper and lower limb was $4 / 5$ with normal tone, sensations intact, left plantar extensor, reflexes normal

MRI brain (plain \& contrast) Showed marked and homogenously enhancing extra-axial mass lesion in right high frontal parasagittal location associated with mass effect midline shift, anterior $2 / 3$ rd of superior sagittal sinus invasion and calvarial hyperostosis, likely meningioma. Thin enhancing subgaleal soft tissue overlying, calvarial hyperostosis- suggestive of transcalvarial infiltration [Figure 1].

Craniotomy and excision of the lesion were done. Intraoperatively right para saggital extra-axial tumor was seen in the frontal lobe. It was hard in consistency. No clear boundary was made out between tumor and brain. The tumor was abutting the sagittal sinus and part of sinus had to be excised. En mass excision was done and sent for histopathology. Postoperative period was uneventful. Subsequent Post-operative resonance imaging (MRI) showed adequate excision along with a resolution of mass effect [Figure 2]. Histology Sections showed glial tissue with suppurative granulomatous inflammation composed of neutrophils, lymphocytes, histiocytes, plasma cells, and multinucleated giant cells. Entangled masses of pigmented fungal hyphae were seen with Splender-Hopple phenomenon and marked fibrosis [Figure 3A,B].GMS stain highlighted colonies shows slender branching fungal hyphae[Figure 3C]. Immunohistochemistry for EMA and Ki67 was done to rule out coexisting meningioma and were negative. Specimen was also send for culture, but no growth was found. Based on the histomorphology, diagnosis of Eumycetoma was made. The patient was started on anti-fungal therapy with Voriconazole. The patient is now doing well at 3 months follow-up. 


\section{DISCUSSION}

Mycetomas are slowly progressive chronic infections of the subcutaneous tissue, usually of the foot and rarely of other parts of the body. The disease was originally reported by Gill in 1842 from Madurai, south India, and Carter in 1860 established its fungal etiology. It is therefore commonly known as Maduramycosis or Madura foot [1]. Common site of infection are extremities, the foot being the most common site of involvement. Cranial involvement has only rarely been reported and occurs due to extension from the nasal sinuses [2-4].

Mycetomas are of three types Eumycetoma caused by true fungi, Actinomycetoma caused by Actinomycetes and Botryomycosis caused by Staphylococcus aureus. The causative agent enters through minor trauma or subcutaneous inoculation. The infection usually starts with a tiny subcutaneous swelling of the foot, which later increase in size, extending into deeper planes and opening to the surface as multiple sinuses discharging viscid, seropurulent fluid containing granules or grains. These are microcolonies of causative agents and their demonstration is of diagnostic value. In actinomycotic mycetoma grains will be composed of very thin filaments, while in mycotic lesions they will be broader and often show septae and chlamydospores. The color and consistency of grains vary. The grains are brown to black in Madurella mycetomi [5].

Cerebral Mycetoma clinically present with Sub-acute or chronic meningitis / Meningoencephalitis. They may rarely present as Intracranial space occupying lesions (ICSOL), parenchymal brain abscesses or granulomas, Skull base syndrome, Vasculitis. and Vascular thrombosis leading to intracranial infarction or hemorrhage.[6] An intracranial abscess is common with certain fungi like Candida, Aspergillus, Phaeohyphomycosis and Zygomycetes spp. Candida and Aspergillus species are commonest. $20 \%-30 \%$ of patients with CNS Aspergillosis had no associated immunosuppression. Candidal abscesses are usually secondary to disseminated disease. CNS Aspergillosis is caused by hematogenous dissemination or direct extension from the paranasal sinus. Zygomycetes (Rhizopus spp., Mucor) generally involve the CNS by direct extension from paranasal sinus. Diabetes mellitus is the most common risk factor. [7]

Diagnosis requires a high degree of clinical suspicion. Brain contrast imaging with MRI or CT is required. Radiologically MRI of fungal abscesses demonstrates a hypointense core with a surrounding iso to mildly hyper-intense rim. T2WI images show increased signal intensity of the core of the lesion with a surrounding rim of hypointensity. Peripheral enhancement is seen on $\mathrm{T} 1$ weighted post contrast enhancement sequence [8].Etiology depends on associated risk factors and ongoing infection in extra-CNS organs. A brain biopsy with cultures may be required [9].

The final diagnosis of eumycetoma was never suspected in our case, preoperatively or even intra-operatively. As it was a pale grain eumycetoma there was absence of purulent material, sulfur granules or a blackish pigmentation. The characteristic sign of pedal eumycetoma "dot in circle' sign was absent. Clinically and radiologically meningioma was suspected. Complete surgical excision of the lesion is the treatment of choice as a response to medical therapy alone is not very promising and is associated with late relapses $[10,11]$.

\section{CONCLUSION:}

In this case, the diagnosis of eumycetoma was never suspected preoperatively or even intra-operatively. Isolated intracranial fungal infection is very rare. Other features contributing to the rarity of this case is that there was no history suggestive of a sinus infection or contact proximity to sinus or contagious spread was not seen. No IV drug abuse or infective foci elsewhere. No signs of granulomatous infection. Eumycetoma of cerebrum is a rare entity. Very few cases were reported in the literature. Infective cause should always be in the differential when analyzing a dural-based lesion with such atypical presentation .

\section{ACKNOWLEDGMENT}

Department of Neurosurgery, Aster MIMS Calicut.

\section{REFERENCES}

[1] Ahmed AO, van Leeuwen W, Fahal A, van de Sande W, Verbrugh H, van Belkum A: Mycetoma caused by Madurella mycetomatis: a neglected infectious burden. Lancet Infect Dis 4:566-574, 2004.

[2] Pollack IF, Pang D, Suchith K E. Chronic granulomatous disease with cranial fungal osteomyelitis and epidural abscess: J. Neusurg. 1986; 67:132-6. [PubMed: 3598663]

[3] Raymond Green T. C, Bolton, Woosley A. B, Chikago ILL. Mycetoma Madura Foot: Annals of Surgery. 1948; 128:1015-21.

[4] Natarajan M, Balakrishnan D, Muthu AK, Arumugham K. Maduromycosis of the brain. Case report. J. Neurosurg. 1975;42:229-231. [PubMed: 1167580]

[5] Sha rma B. S, Khosla V. K, Kak V. K, Banerjee A. K, Path, et al. Intra cranial fungal grnuloma. Surg Neurol. 1997;47:489-7. [PubMed: 9131036]

[6] Hickey B B. Cranial maduromycosis. Trans R Soc Trop Med Hyg. 1950;50:393-6. [PubMed: 13360990]

[7] Phuttharak W, Hesselink JR, Wixom C. MR features of cerebral aspergillosis in an immunocompetent patient: Correlation with histology and elemental analysis. Am J Neuroradiol. 2005;26:835-8. [PubMed 15814930]

[8] Ahmed Munvar, Jyothi sureka, Geetha Chacko, Anu Eapen. MRI findings in cranial eumycetoma. Indian Journal of Rdiolology and Imaging. 2011;21:261-3. [PMCID: PMC3249938] [PubMed: 22223935]

[9] Maheshwari S, Figueiredo A, Narurkar S, Goel A. Madurella mycetoma-A rare case with cranial extension. World Neurosurg. 2010;73:69-71. [PubMed: 20452871]

[10] Welsh O, Salinas MC, Rodríguez MA: Treatment of eumycetoma and actinomycetoma. Curr Top Med Mycol 6:47-71, 1995

[11] Narayanam AS, Kasliwal MK, Suri A, Sharma BS. Eumycetoma presenting as a cerebellopontine angle mass lesion. Clinical Neurology and Neurosurgery. 2007; 109:516-9. [PubMed: 17449170] 


\section{AUTHORS}

1. Dr. Mahsheena K M, Specialist Pathologist, Aster MIMS, Calicut, Kerala. India. 2. Dr. Lilly Rajeevan, Chief and Senior Consultant Pathologist, Aster MIMS, Calicut, India.

3. Dr. Shalini Kuruvila, Senior Pathologist, Aster MIMS, Calicut, Kerala. India.
4. Dr. Kavitha K P, Senior Pathologist, Aster MIMS, Calicut, Kerala. India.

Correspondence Author - Dr. Mahsheena.k.m

Kalpaka House, Koduvally P.O, Calicut, Kerala state, India. Phone numbers: 8547200435. Email-mahshikm@yahoo.com.

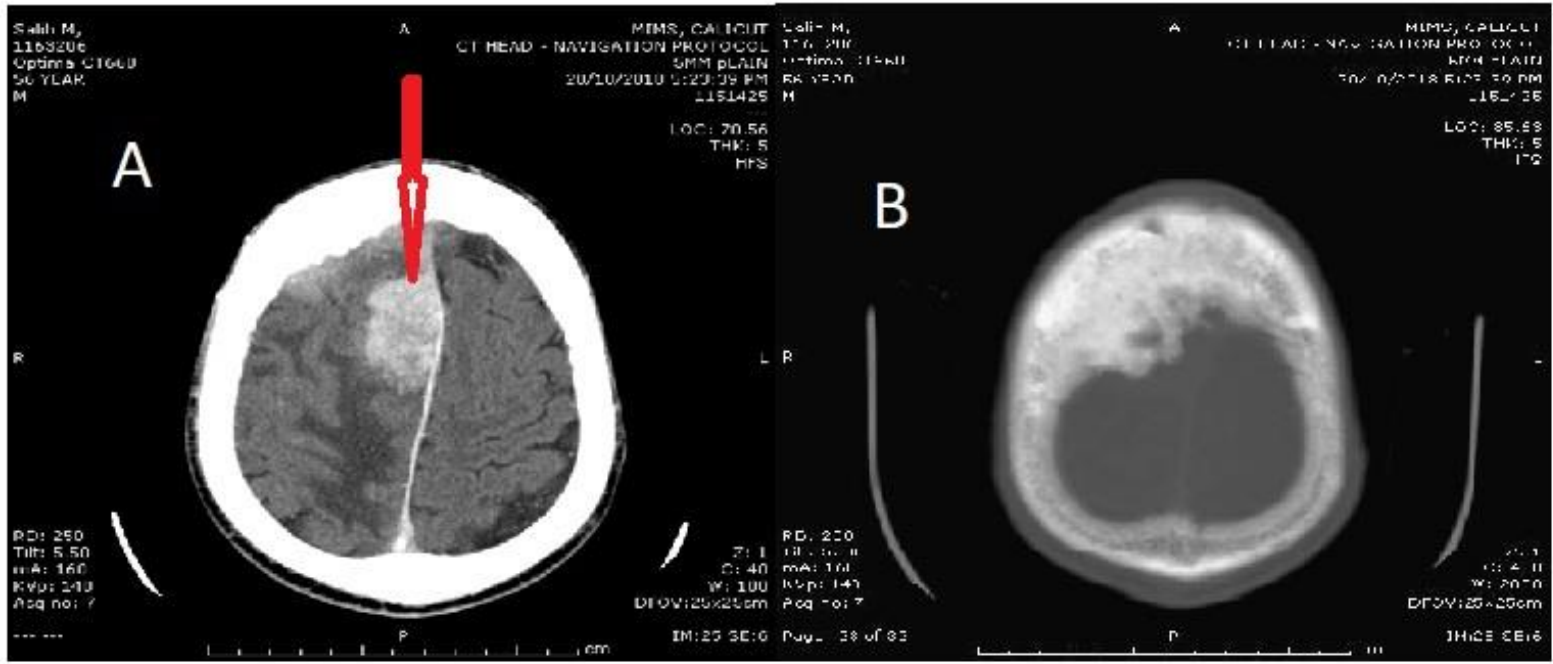

Fig No:1- Contrast enhanced CT scan of the brain shows A)homogenously enhancing hyper dense lesion in the right frontal region(arrow), Para sagittal location with adjacent edema B) Hyperostosis of the overlying frontal bone

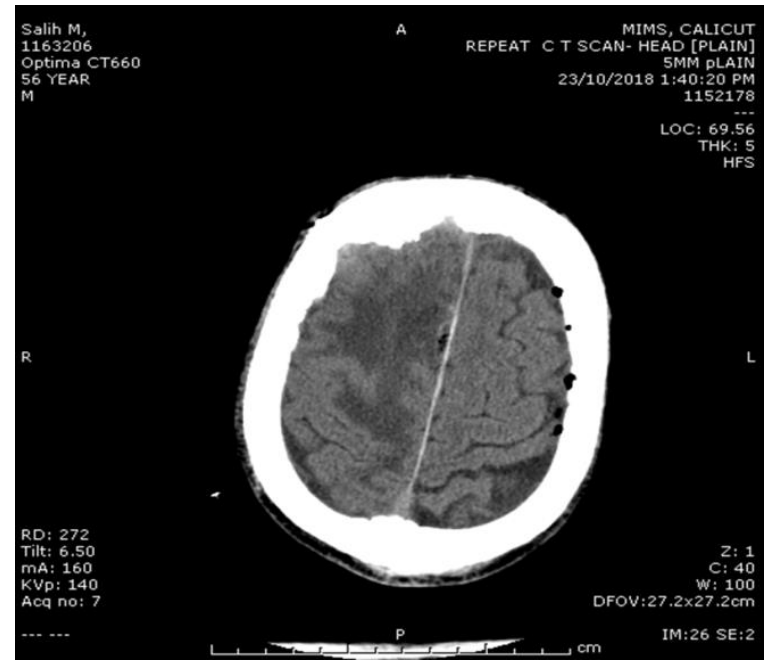

Fig No: 2-Post operative imaging (CT) showed adequate excision along with a resolution of mass effect 


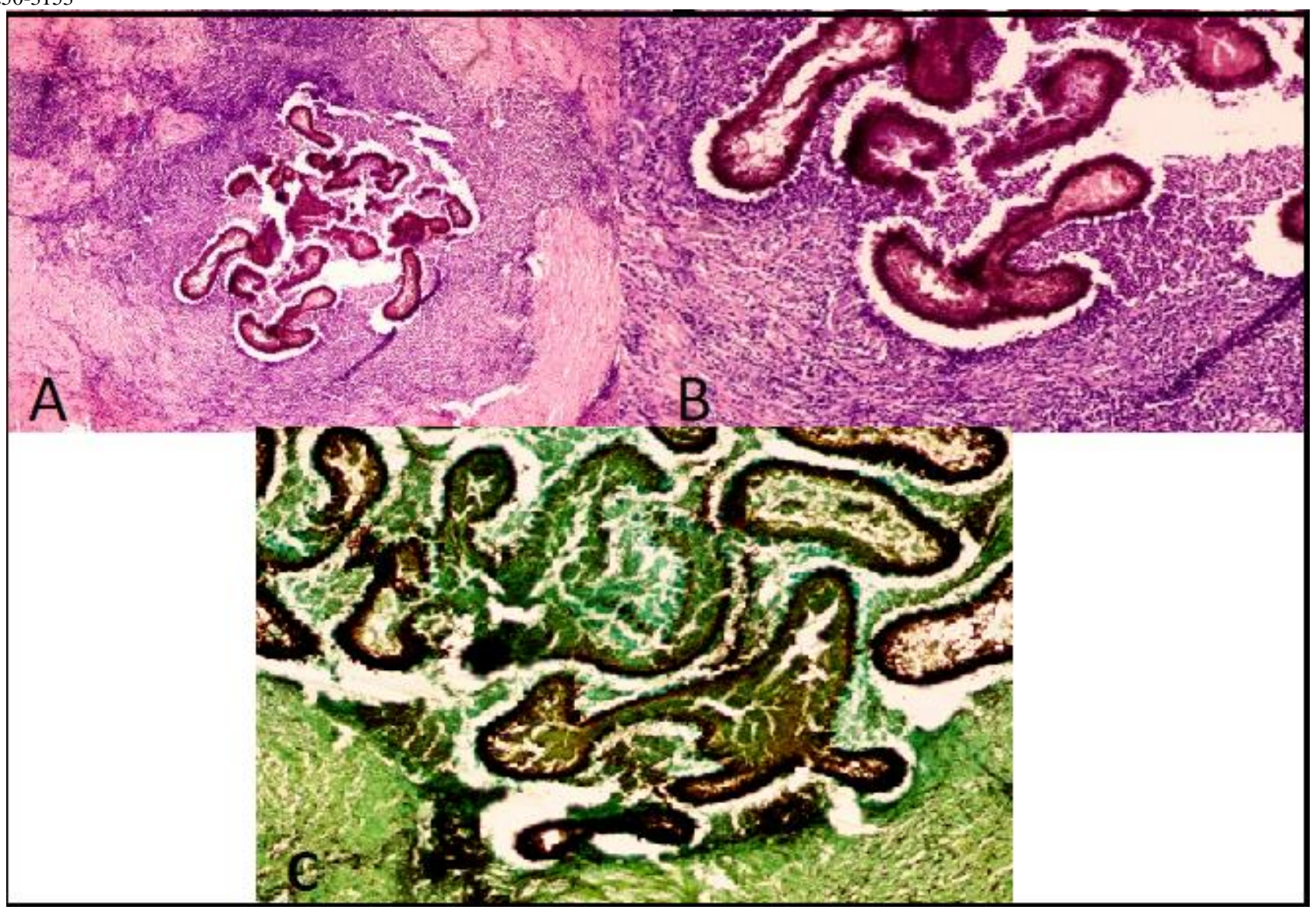

Fig No: 3 Photomicrography of glial tissue A \& B)with entangled masses of pigmented fungal hyphae with Splender-Hopplei phenomenon and marked fibrosis.(H\&E, 100x,400x) C) GMS stain highlighted colonies shows slender branching fungal hyphae. (GMS, 400x) 